Published in Chemical Physics Letters 383: 584-591, 2004

\title{
Hybrid ligand-field theory/quantum chemical calculation of the fine structure and ZFS in lanthanide(III) complexes
}

\author{
Alain Borel ${ }^{\mathrm{a}}$, Lothar Helm ${ }^{\mathrm{a}}$, Claude A.E. Daul ${ }^{\mathrm{b}, *}$

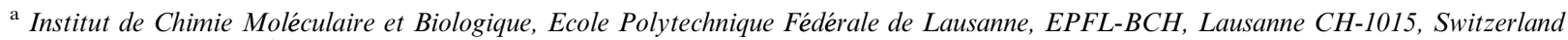 \\ ${ }^{\mathrm{b}}$ Département de Chimie, Université de Fribourg, Pérolles, Fribourg CH-1700, Switzerland
}

Received 8 September 2003; in final form 4 November 2003

\begin{abstract}
Ligand field effects in lanthanide ions compounds have consequences for optical and magnetic spectroscopy. In the analysis of electron paramagnetic resonance spectra of $\mathrm{Gd}^{3+}$ complexes, a major role is played by the zero field splitting (ZFS), which is a high order consequence of the ligand field and the spin-orbit coupling. We present a general parameterized method and a computer program for the study of the excited states of lanthanide complexes. We apply it for the first principles determination of the ZFS of the $\left[\mathrm{Gd}(\mathrm{H} 2 \mathrm{O})_{8}\right]^{3+}$ aqua ion and the corresponding EPR peak-to-peak width in solution. We calculate the influence of the various contributions to the effective Hamiltonian on the splitting of the ground state multiplet.
\end{abstract}

\section{Introduction}

In recent years considerable amount of work has been performed studying the properties of paramagnetic Gadolinium(III) $(S=7 / 2)$ in solution. This interest originates from the strong NMR-relaxation enhancement of neighboring water protons that they induce. Complexes of $\mathrm{Gd}^{3+}$ are therefore widely used as contrast agents in medical magnetic relaxation imaging (MRI) [1].

To get a better understanding of the influence of the electron spin relaxation on the ${ }^{1} \mathrm{H}$-relaxation enhancement a general theory of the relaxation of an $\mathrm{S}$ state paramagnetic metal ion in solution was developed $[2,3]$. The basic idea in this model is that electron spin relaxation of $\mathrm{Gd}^{3+}$ is governed by the combined effects of a transient zero field splitting (ZFS) and a static ZFS originating from the mean ligand field of surrounding atoms. The ZFS Hamiltonian is expressed as a sum of linear combinations of irreducible rank- $k$ tensor operators with real coefficients, $B_{k \eta}$, which determine the

\footnotetext{
${ }^{*}$ Corresponding author. Fax: +41-26-3009738.

E-mail address: claude.daul@unifr.ch (C.A.E. Daul).
}

magnitude of each contribution [3]. Unfortunately, EPR in solution does not give access the individual coefficients $B^{k \alpha}$ (where $\alpha$ designates linearly independent contributions of decreasing symmetry) but only to parameters which are the roots of the sum over $\alpha$ of $\left(B^{k \alpha}\right)^{2}$.

To get deeper insight a theoretical calculation of electronic fine structure is needed. The magnetic and spectroscopic properties of the lanthanide ions depend on the $\mathrm{f}$ electron structure, which is generally understood in the framework of a model where the f orbitals are considered shielded from the chemical environment. In this model the ground and excited states essentially arise from the electrostatic repulsion between the $\mathrm{f}$ electrons (for a configuration $\mathrm{f}^{n}$ where $n>1$ ) and the spin-orbit coupling with the angular momentum of the f orbitals (quantum number $l=3$ ). Therefore they can be conveniently labeled according to their electron spin $S$, orbital angular momentum $L$ and total angular momentum $J$.

Further splitting of the spectroscopic states under the influence of the ligand field can be observed in the luminescence spectra, as observed in a number of studies. The ligand field does also affect the magnetic properties. The ZFS of the ${ }^{8} \mathrm{~S}_{7 / 2}$ ground state of the $\mathrm{f}^{7}$ ion $\mathrm{Gd}^{3+}$ is evident in the solid state [4-6], and its modulation is the 
origin of the electron spin relaxation in solution as described in $[2,3]$.

The ligand field effects in lanthanide compounds have been described in the past using a number of phenomenological approaches [7], such as the simple pointcharge model, the angular overlap model (AOM) of Jørgensen and co-worker [8,9] and more recently the superposition model [10]. There has been much confusion in the literature between the conventional ligand field Hamiltonian and the so-called crystal field of the spin Hamiltonian (i.e., ZFS) due to a similar formalism and terminology [11]. A rigorous calculation of the molecular energy levels should help us build an interface between the two descriptions.

We present here a general method for the ab initio calculation of the excited states of lanthanide(III) complexes. In this approach, the complete active space of the $4 \mathrm{f}^{n} \rightarrow 4 \mathrm{f}^{n}$ excitations is used to perform a configuration interaction (CI) calculation in a basis set of single Slater determinants. Therefore, the method is not restricted to calculations within a defined ${ }^{S} L_{J}$ multiplet and the $J$-mixing effect of the ligand field can be treated naturally. The Hamiltonian is calculated using a few simple parameters that can be either extracted from experimental data for a semi-empirical calculation or calculated by standard quantum mechanical methods for a complete $a b$ initio study. We demonstrate the applicability of this approach with the example of the $\mathrm{Gd}^{3+}$ octa aqua ion where the $\mathrm{f}^{7}$ configuration leads to the largest microstate basis set (3432 single determinants) and the high symmetry of the coordination polyhedron lends itself to a detailed analysis of the spin Hamiltonian matrix.

\section{Theoretical section}

Besides the central potential of the nucleus, the general Hamiltonian acting upon atomic metal orbitals may be written as

$\hat{H}=\hat{H}_{\mathrm{ER}}+\hat{H}_{\mathrm{SO}}+\hat{H}_{\mathrm{LF}}$,

where the three terms correspond to the inter-electron repulsion, the spin-orbit coupling and the ligand field, respectively. The matrix elements for each of these operators can be expressed in a basis of single Slater determinants $|\Psi|=\left|\psi_{1} \psi_{2} \psi_{3} \cdots \psi_{n}\right|$ where $\psi_{i}$ is a singleoccupied spin orbital and $n$ is the number of $f$ electrons. The matrix elements are generally defined in a basis of spin orbitals and Slater's rules [12,13] allow a straightforward calculation for single determinants.

The number of determinants to consider is $C_{N}^{n}=\frac{N !}{(N-n) ! n !}$ for $n$ electrons in $N$ spin orbitals. For $\mathrm{f}$ orbitals, the number of determinants is between 14 ( $\mathrm{f}^{1}$ or $\left.\mathrm{f}^{9}\right)$ and $3432\left(\mathrm{f}^{7}\right)$. Several physical parameters, defined later in the text, are also involved in the calculation of the
Hamiltonian matrix elements. All of these parameters can be calculated quantum mechanically if required.

The respective matrix elements of $H_{\mathrm{ER}}, H_{\mathrm{SO}}$ and $H_{\mathrm{LF}}$ are given next.

$$
\begin{aligned}
\left\langle\Phi\left|\hat{H}_{\mathrm{ER}}\right| \psi\right\rangle & =\sum_{g, h, i, j=1}^{n} A_{\mathrm{ER}}^{g h i j}\left\langle\varphi_{g} \varphi_{h}\left|\hat{H}_{\mathrm{ER}}\right| \psi_{i} \psi_{j}\right\rangle \\
& =\sum_{g, h, i, j=1}^{n} \sum_{k=0,2,4,6} A_{\mathrm{ER}}^{g h i j} C(k, g, h, i, j) F_{k} .
\end{aligned}
$$

The matrix elements of $\hat{H}_{\mathrm{ER}}$ can be written as linear combinations of a limited number of two-electron integrals. For $\mathrm{d}$ electrons, these are the three well-known Racah parameters $A, B$ and $C$, or Slater-Condon integrals $F_{k}, k=0,2,4$. For $\mathrm{f}$ electrons, one may use either the four $E^{n}$ parameters of Racah $(n=0,1,2,3)$, or Slater-Condon integrals $(k=0,2,4,6)$. The real coefficients $A_{\mathrm{ER}}$ combine the Coulomb and exchange matrix elements in an orbital basis set according to Slater's rules. The $C(k, g, h, i, j)$ are products of the vector coupling coefficients for real spherical harmonics [14]. Since our basis set is defined using $\mathrm{f}^{n}$ micro-states, it is convenient to express the electrostatic repulsion matrix elements using four two-electrons integrals involving $\mathrm{f}$ spin orbitals:

$$
\begin{aligned}
G_{0}= & \left\langle\mathrm{f}\left[x\left(3 x^{2}-y^{2}\right)\right] \mathrm{f}\left[x\left(3 x^{2}-y^{2}\right)\right]\right| \mathrm{f}\left[x\left(3 x^{2}-y^{2}\right)\right] \\
& \left.\times \mathrm{f}\left[x\left(3 x^{2}-y^{2}\right)\right]\right\rangle \\
= & F_{0}+25 F_{2}+9 F_{4}+463 F_{6}, \\
G_{2}= & \left\langle\mathrm{f}\left[x\left(3 x^{2}-y^{2}\right)\right] \mathrm{f}\left[z\left(y^{2}-z^{2}\right)\right]\right| \mathrm{f}\left[x\left(3 x^{2}-y^{2}\right)\right] \\
& \left.\times \mathrm{f}\left[z\left(y^{2}-z^{2}\right)\right]\right\rangle \\
= & 12.5 F_{2}+15 F_{4}+234.5 F_{6}, \\
G_{4}= & \left\langle\mathrm{f}\left[z\left(y^{2}-z^{2}\right)\right] \mathrm{f}\left[z\left(y^{2}-z^{2}\right)\right]\right| \mathrm{f}\left[z\left(y^{2}-z^{2}\right)\right] \\
& \left.\times \mathrm{f}\left[z\left(y^{2}-z^{2}\right)\right]\right\rangle \\
= & F_{0}+84 F_{4}+288 F_{6}, \\
G_{6}= & \left\langle\mathrm{f}\left[z\left(y^{2}-z^{2}\right)\right] \mathrm{f}\left[z\left(y^{2}-z^{2}\right)\right] \mid \mathrm{f}\left[x\left(3 x^{2}-y^{2}\right)\right] \mathrm{f}\left[y z^{2}\right]\right\rangle \\
= & -27.111 F_{4}+162.666 F_{6} .
\end{aligned}
$$

The linear coefficients above can be derived from the corresponding Clebsch-Gordan series. A sample calculation on the $\mathrm{f}^{2}$ configuration, using the known energies of the spectroscopic terms as functions of the SlaterCondon parameters (see for example Dieke [15]) was performed to check the calculated coefficients. The twoelectron integrals can be calculated ab initio, or fitted to experimental data. The overestimation of the SlaterCondon parameters by Hartree-Fock calculations on the free ions [16] by some $40 \%$ compared to the aqua ions measurements [17] leads us to prefer the experimental parameters for pragmatic reasons. For comparison purposes, we also calculated the electron repulsion 


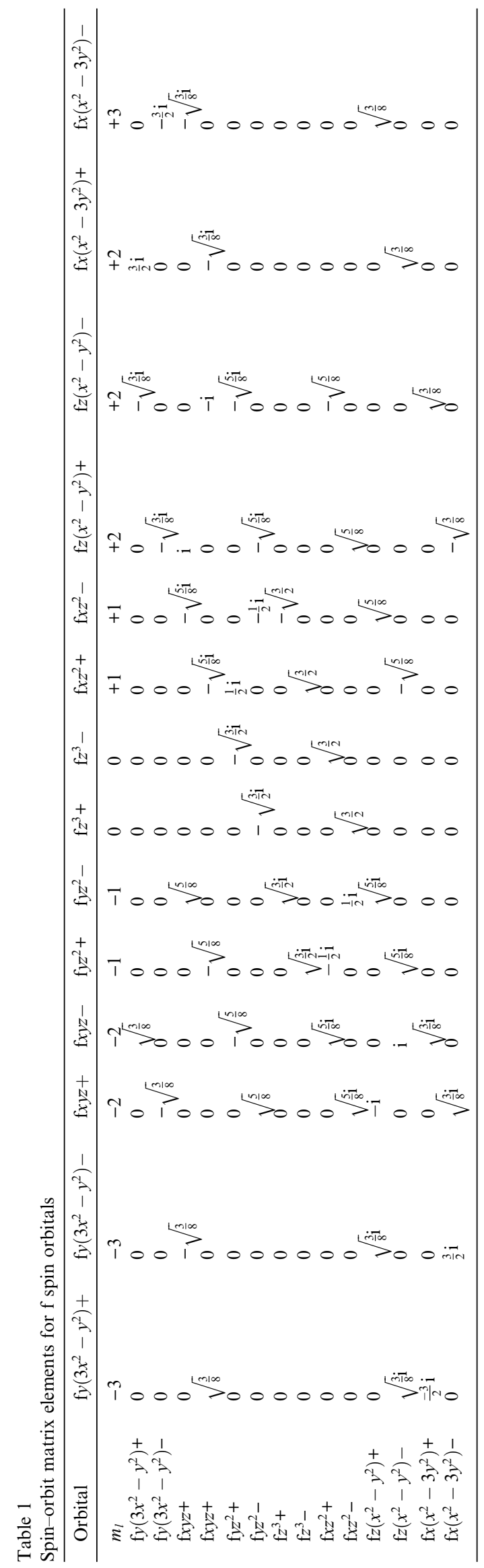

parameters $G_{0}$ to $G_{6}$ using the ligand field density functional theory (LFDFT) method $[18,19]$.

The spin-orbit coupling elements are readily calculated from the individual terms for each spin orbital using the spin and angular momentum operators $\left(\hat{L}_{z}, \hat{L}_{x}=\right.$ $\left.\frac{1}{2 i}\left(\hat{L}_{+}+\hat{L}_{-}\right), \hat{L}_{y}=\frac{1}{2 i}\left(\hat{L}_{+}-\hat{L}_{-}\right)\right)$defined in a basis of real spherical harmonics of order $l=3$ for $\mathrm{f}$ electrons:

$$
\left\langle\Phi\left|\hat{H}_{\mathrm{SO}}\right| \psi\right\rangle=\zeta \sum_{i, j=1}^{n} A_{\mathrm{SO}}^{i j}\left\langle\varphi_{i}|\hat{l} \hat{s}| \psi_{j}\right\rangle
$$

where $\zeta$ is the spin-orbit coupling constant and the matrix elements $\left\langle\varphi_{i}|\hat{l} \hat{s}| \psi_{j}\right\rangle$ are given in Table 1 . We can calculate $\zeta=\left\langle\frac{1}{r} \frac{\mathrm{d} V}{\mathrm{~d} r}\right\rangle$ from a DFT calculation of the electronic structure of the free $\mathrm{Gd}^{3+}$ ion.

Finally, the ligand field terms are described by linear combinations of the matrix elements of the effective ligand field potential $\hat{V}_{\mathrm{LF}}$ acting upon the f-orbitals. The $7 \times 7$ matrix is reduced to a set of 28 independent matrix elements by the Hermicity of the ligand field Hamiltonian.

$$
\left\langle\Phi\left|\hat{H}_{\mathrm{LF}}\right| \psi\right\rangle=\sum_{i=1}^{7} \sum_{j=1}^{i} A_{\mathrm{LF}}^{i j}\left\langle\mathrm{f}_{i}\left|\hat{V}_{\mathrm{LF}}\right| \mathrm{f}_{j}\right\rangle .
$$

\section{Computational section}

A computer program implementing Eqs. (2)-(4) was written in the MATLA B [20] programming language. The program calculates the matrix elements of the Hamiltonian from the following parameters:

1. Four electron repulsion parameters, that can be Racah or Slater-Condon parameters, which we convert to the four two-electron integrals described above. The matrix coefficients $C(k, g, h, i, j)$ for an arbitrary $l^{n}$ configuration are calculated by a standalone Matlab or Fortran program.

2. The spin-orbit coupling constant.

3. 28 ligand field matrix elements.

The experimental Racah $E^{n}$ parameters $(0,5761$, 28.02 and $582 \mathrm{~cm}^{-1}$ for $n=0,1,2,3$, respectively) and spin-orbit coupling constant $\xi\left(1450 \mathrm{~cm}^{-1}\right)$ were taken from the extensive work of Carnall et al. [17] on the lanthanide(III) aqua ions.

The $\mathrm{f}$ ligand field matrix elements were obtained from the occupied $f$ spin orbitals energies of $\left[\mathrm{Gd}\left(\mathrm{H}_{2} \mathrm{O}\right)_{8}\right]^{3+}$ as calculated by the density functional theory (DFT) program ADF 2001 [21]. The structure of the complex was optimized in the $\mathrm{D}_{4 \mathrm{~d}}$ symmetry following Schafer and Daul [22], using an unrestricted triple-zeta basis set with polarization functions and a relativistic effective core potential (Gd: Cd core, O: He core). Becke [23] and Perdew [24] non-local corrections were used. The 7 molecular orbitals with dominant $4 \mathrm{f}$ character were projected onto the reduced basis set of the atomic $\mathrm{f}$ orbitals and the matrix elements of $V_{\mathrm{LF}}$ were calculated 
from the Kohn-Sham molecular orbitals energies $\varepsilon^{\mathrm{KS}}$ and projected coefficients $c_{\mu}=\left\langle\mathrm{f}_{\mu} \mid \phi^{\mathrm{KS}}\right\rangle$ [18].

$\hat{V}_{\mathrm{LF}}=\hat{C} \hat{E} \hat{C}^{-1}$,

so that

$\left\langle\mathrm{f}_{\mu}\left|\hat{V}_{\mathrm{LF}}\right| \mathrm{f}_{v}\right\rangle \approx \sum_{i=1}^{7} c_{\mu i} c_{v i} \varepsilon_{i}^{\mathrm{KS}}$.

Although the Kohn-Sham energies $\varepsilon^{\mathrm{KS}}$ also involve the electron repulsion, Atanasov and Rauzy [25] observed that the $V_{\mathrm{LF}}$ matrix elements calculated from the full manifold of Slater determinants were essentially the same as those obtained using this simple method.

The ab initio spin-orbit coupling constant was calculated using the ХАтом program [26]. Two-electron integrals were obtained using the LFDFT method and SD energies calculated with ADF for the optimized structure.

The eigenvalues and eigenvectors of the Hamiltonian were calculated with and without ligand field contribution, and sorted in ascending order. The total calculation time for the $\mathrm{f}^{7}$ configuration requires a substantial amount of memory ( $1 \mathrm{~Gb}$ of RAM and about $3 \mathrm{~Gb}$ of mass storage) but takes only a few of hours on a modern workstation.

\section{Results}

\subsection{DFT results}

The program ХАтом was used to numerically solve the Kohn-Sham equations for the free $\mathrm{Gd}^{3+}$ ion and calculate the spin-orbit coupling constant. The resulting value $\left(1283 \mathrm{~cm}^{-1}\right)$ is consistent with the experimental value reported by Carnall $\left(1450 \mathrm{~cm}^{-1}\right)$.

The optimized $\mathrm{D}_{4 \mathrm{~d}}$ structure is shown in Fig. 1. The calculated orbital energies around the barycenter are reported in Table 2.

Following the LFDFT method, the optimized geometry was used to obtain a spin-restricted average of configurations (AOC) and the 3432 Slater determinants energies were calculated taking advantage of the proven ADF [21] code. The two-electron integrals $G_{0}, G_{2}, G_{4}$ and $G_{6}$ were fitted to the Slater determinants energies. The results correspond to the following Racah parameters: $E^{2}=4586.6 \mathrm{~cm}^{-1}, E^{3}=34.2 \mathrm{~cm}^{-1}$ and $E^{3}=767.8 \mathrm{~cm}^{-1}$. The corresponding value $E^{1}\left(4.78 \times 10^{6} \mathrm{~cm}^{-1}\right)$ can be arbitrarily set to zero since its only effect will be to shift the multiplet energies by the same amount. These parameters are in general agreement with those of Carnall $(0,5761$, 28.02 and $582 \mathrm{~cm}^{-1}$ ). One possible origin for the remaining discrepancy is the numerical evaluation of integrals in the framework of DFT, which is difficult to achieve with very high accuracy.

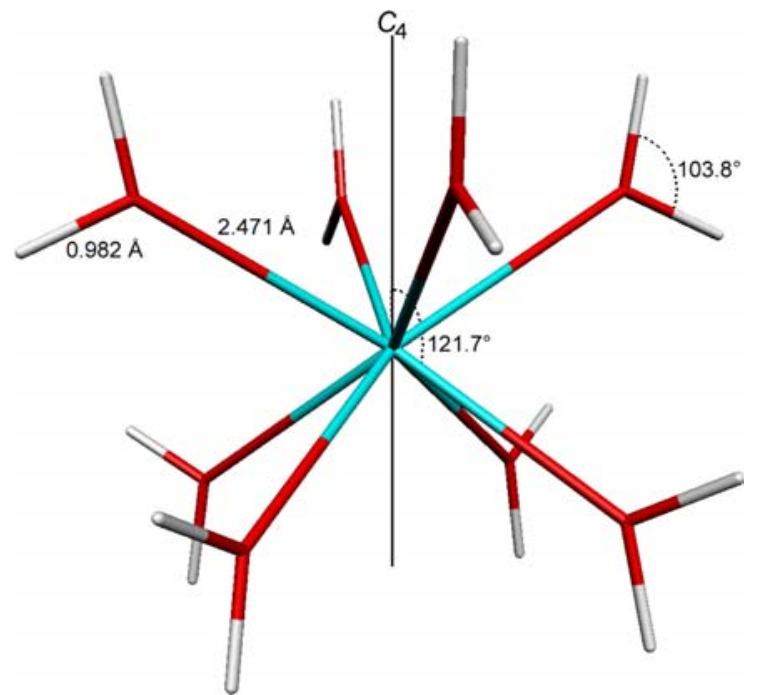

Fig. 1. DFT-optimized structure of $\left[\mathrm{Gd}\left(\mathrm{H}_{2} \mathrm{O}\right)_{8}\right]^{3+}$ in the $\mathrm{D}_{4 d}$ symmetry.

Table 2

f spin orbitals splitting in $\left[\mathrm{Gd}\left(\mathrm{H}_{2} \mathrm{O}\right)_{8}\right]^{3+}$ calculated by NLDA DFT

\begin{tabular}{lllrl}
\hline Representation & ADF FO & Orbital & $m_{l}$ & Energy (eV) \\
\hline $\mathrm{e}_{1}$ & $F: z 2 x$ & $\mathrm{f} x z^{2}$ & +1 & -0.07071 \\
$\mathrm{e}_{1}$ & $F: z 2 y$ & $\mathrm{f} y z^{2}$ & -1 & -0.07071 \\
$\mathrm{e}_{3}$ & $F: y$ & $\mathrm{f} y\left(3 x^{2} y^{2}\right)$ & -3 & -0.00071 \\
$\mathrm{e}_{3}$ & $F: x$ & $\mathrm{f} x\left(x^{2}-3 y^{2}\right)$ & +3 & -0.00071 \\
$\mathrm{~b}_{2}$ & $F: z 3$ & $\mathrm{f} z^{3}$ & 0 & 0.040286 \\
$\mathrm{e}_{2}$ & $F: x y z$ & $\mathrm{f} x y z$ & -2 & 0.051286 \\
$\mathrm{e}_{2}$ & $F: z$ & $\mathrm{f} z\left(y^{2} z^{2}\right)$ & +2 & 0.051286 \\
\hline
\end{tabular}

\subsection{Excited states energies}

The method presented in the theoretical section was applied to the calculation of the energy levels of the free $\mathrm{Gd}^{3+}$ ion $\left(\hat{H}=\hat{H}_{\mathrm{ER}}+\hat{H}_{\mathrm{SO}}, \hat{H}_{\mathrm{LF}}=0\right)$ using the experimental parameters of Carnall. The energies of the first states up to the ${ }^{6} \mathrm{D}$ multiplet are reported in Table 3 with their multiplicity and assignment. The multiplicity with and without spin-orbit coupling is used to perform the assignment of the generated levels. We can use the apparent splitting of the ${ }^{8} S_{7 / 2}$ free ion ground state, which should be zero, to estimate the magnitude of the numerical errors induced by the Hamiltonian diagonalization. In our calculation the error is close to $10^{-2} \mathrm{~cm}^{-1}$.

Turning on the ligand field interaction, most of the states are split into Kramers doublets. The fundamental ${ }^{8} \mathrm{~S}$ multiplet is split into 4 doublets with relative energies $0,0.03,0.084$ and $0.142 \mathrm{~cm}^{-1}$. The splitting of the excited states is larger and makes the assignment more difficult due to a strong overlap between the states originating from different multiplets. Our ab initio spinorbit coupling and electron repulsion parameters lead to a larger splitting $\left(0,0.292,0.657\right.$ and $\left.1.129 \mathrm{~cm}^{-1}\right)$. This is clearly excessive as the experimental splitting of the ${ }^{8} \mathrm{~S}$ 
Table 3

Energy and degeneracy of the first eigenstates of the Hamiltonian (free ion)

\begin{tabular}{ccl}
\hline Multiplicity & Energy $\left(\times 10^{3} \mathrm{~cm}^{-1}\right)$ & Assignment \\
\hline 8 & 0.00 & ${ }^{8} \mathrm{~S}_{7 / 2}$ \\
8 & 31.18 & ${ }^{6} \mathrm{P}_{7 / 2}$ \\
6 & 31.75 & ${ }^{6} \mathrm{P}_{5 / 2}$ \\
4 & 32.32 & ${ }^{6} \mathrm{P}_{3 / 2}$ \\
8 & 33.65 & ${ }^{6} \mathrm{I}_{7 / 2}$ \\
10 & 34.01 & ${ }^{6} \mathrm{I}_{9 / 2}$ \\
18 & 34.22 & ${ }^{6} \mathrm{I}_{17 / 2}$ \\
12 & 34.31 & ${ }^{6} \mathrm{I}_{11 / 2}$ \\
14 & 34.49 & ${ }^{6} \mathrm{I}_{13 / 2}$ \\
16 & 34.50 & ${ }^{6} \mathrm{I}_{15 / 2}$ \\
10 & 38.36 & ${ }^{6} \mathrm{D}_{9 / 2}$ \\
2 & 39.22 & ${ }^{6} \mathrm{D}_{1 / 2}$ \\
8 & 39.36 & ${ }^{6} \mathrm{D}_{7 / 2}$ \\
4 & 39.48 & ${ }^{6} \mathrm{D}_{3 / 2}$ \\
6 & 39.64 & ${ }^{6} \mathrm{D}_{5 / 2}$ \\
\hline
\end{tabular}

ground state of the $\mathrm{Gd}^{3+}$ aqua ion is between 0.2 and $0.5 \mathrm{~cm}^{-1}[2,4,27]$. We feel more confident using the semiempirical splitting as a basis for further analysis. Unless otherwise specified, the latter is systematically used in the following discussion.

\subsection{ZFS parameters for the electron spin relaxation in solution}

In $\mathrm{D}_{4 \mathrm{~d}}$ symmetry, the ZFS spin Hamiltonian acting on the $S=7 / 2$ multiplet can be written as [3]:

$\hat{H}=\sum_{k=2,46} B^{k} \hat{T}_{0}^{k}$,

where $\hat{T}_{0}^{k}$ is an spherical spin tensor [28] and $B^{k}$ is the corresponding spin Hamiltonian parameter. The $T_{0}^{k}$ tensors are diagonal in a basis of $\left|S, m_{S}\right\rangle$ spin states. Therefore, the eigenvalues of this spin Hamiltonian (which we can identify with the eight lower energies of our CI calculation) are linear combinations of the $B^{k}$ parameters. Following perturbation theory, we can assume that the second order term $B^{2} T_{0}^{2}$ dominates the effect and write the following set of linear equations:

$$
\begin{aligned}
& 8.5732 B^{2}+25.0998 B^{4}+20.7255 B^{6}+C=0.142 \mathrm{~cm}^{-1}, \\
& 1.2247 B^{2}-46.6139 B^{4}-103.6274 B^{6}+C=0.084 \mathrm{~cm}^{-1}, \\
& -3.6742 B^{2}-10.7571 B^{4}+186.5293 B^{6}+C=0.032 \mathrm{~cm}^{-1}, \\
& -6.1237 B^{2}+32.2712 B^{4}-103.6274 B^{6}+C=0 \mathrm{~cm}^{-1},
\end{aligned}
$$

where the numerical coefficients are functions of $S$ and $m_{S}$ readily obtained from the tensor definitions of Buckmaster et al. [28]. Solving this set of linear equations leads to the values reported in Table 4 .

The calculation of the EPR peak-to-peak width, representative of the transverse spin relaxation rate, can be performed using these parameters (in this case $a_{k}=\left|B^{k}\right|$ ) and a second rotational correlation time of
Table 4

ZFS parameters assuming a dominant second order contribution

\begin{tabular}{cccl}
\hline$k$ & $B^{k}\left(\mathrm{~cm}^{-1}\right)$ & $B^{k}\left(\times 10^{10} \mathrm{~s}^{-1}\right)$ & $a_{k}\left(\times 10^{10} \mathrm{~s}^{-1}\right)$ \\
\hline 2 & 0.0095 & 0.1791 & 0.1791 \\
4 & -0.0002 & -0.0038 & 0.0038 \\
6 & 0 & 0 & 0 \\
\hline
\end{tabular}

35 ps [27]. At room temperature and $9.425 \mathrm{GHz}$ microvawe frequency (X-band EPR, where rotational modulation of the ZFS essentially determines the electron spin relaxation), we obtain a peak-to-peak width $\Delta H_{\mathrm{pp}}=26 \mathrm{G}$.

\section{Discussion}

The calculated geometry (Fig. 1) seems reasonable. The $\mathrm{Gd}-\mathrm{O}$ distance $(2.471 \AA$ ) is similar to the reported, 2.4-2.6 A, experimental [29] and theoretical values $[22,30]$. Using the same method (GGA DFT), Schafer and Daul [22] obtained $2.52 \AA$, significantly longer than our result. GGA was known to produce too long bonds when metals where involved. The improved basis sets in recent versions of ADF appear to solve this problem.

The calculated energies of the ER/SO multiplets (Table 2) are in semi-quantitative agreement with the experimental optical data of Carnall et al. [17]. However the energy calculations of Carnall also included configuration interaction terms [31-33] such as $4 \mathrm{f}^{n} \rightarrow 4 \mathrm{f}^{n-1} 5 \mathrm{~s}^{1}$ or $4 \mathrm{f}^{n-1} 5 \mathrm{~d}^{1}$ excitations that are not taken into account in our present work. Our Hamiltonian spans the whole active space of the $\mathrm{f}^{n}$ configurations and it is expected that our DFT calculation (correlation effects) of the ligand field parameters will approximately represent the contribution of these extra terms.

The total splitting of the ${ }^{8} \mathrm{~S}$ fundamental multiplet $\left(0.142 \mathrm{~cm}^{-1}\right)$ agrees in magnitude with the experimental EPR data in the solid state [4] $\left(0.25 \mathrm{~cm}^{-1}\right)$ and in aqueous solution [2,27] $\left(0.38-0.46 \mathrm{~cm}^{-1}\right)$. However, it appears too low compared with the experimental results.

We performed several subsequent calculations to study the influence of the three interactions on the relative energies of the lower eight eigenstates of the Hamiltonian. The results are reported in Tables 5-7, together with the detailed results obtained by Bleaney and co-worker [4] for $\mathrm{Gd}^{3+}$ doped into a solid lanthanum ethylsulfate matrix.

The effect of the spin-orbit coupling (Table 5) is rather important. The total splitting of the multiplet is $50 \%$ larger $\left(0.2149\right.$ instead of $\left.0.142 \mathrm{~cm}^{-1}\right)$ with a $10 \%$ increase of the coupling constant. The splitting in this case is actually in significantly better agreement with the values of Bleaney. However, the SO coupling constant should not be seen as the only source of error here, as even $\xi=1600 \mathrm{~cm}^{-1}$ would lead to an excessive spinorbit splitting of the excited states [34]. 
Table 5

Influence of the spin-orbit coupling on the ZFS

\begin{tabular}{llllll}
\hline Reference & Bleaney & 0.5 SO & 0.9 SO & 1.1 SO & 1.5 SO \\
\hline 0.000 & 0 & 0.0000 & 0.0000 & 0.0000 & 0.0000 \\
0.000 & 0 & 0.0000 & 0.0001 & 0.0481 & 0.0001 \\
0.032 & 0.049 & 0.0044 & 0.0205 & 0.0482 & 0.1670 \\
0.032 & 0.049 & 0.0044 & 0.0205 & 0.1310 & 0.1670 \\
0.084 & 0.132 & 0.0049 & 0.0500 & 0.1311 & 0.5173 \\
0.084 & 0.132 & 0.0049 & 0.0500 & 0.2148 & 0.5175 \\
0.142 & 0.245 & 0.0169 & 0.0886 & 0.2149 & 0.7595 \\
0.142 & 0.245 & 0.0169 & 0.0887 & 0.7596 \\
\hline
\end{tabular}

Table 6

Influence of the electrostatic repulsion on the ZFS

\begin{tabular}{llllll}
\hline Reference & Bleaney & $0.5 \mathrm{ER}$ & $0.9 \mathrm{ER}$ & $1.1 \mathrm{ER}$ & $1.5 \mathrm{ER}$ \\
\hline 0.000 & 0 & 0.0000 & 0.0000 & 0.0000 & 0.0000 \\
0.000 & 0 & 0.0002 & 0.0001 & 0.0001 & 0.0001 \\
0.032 & 0.049 & 0.1888 & 0.0417 & 0.0264 & 0.0191 \\
0.032 & 0.049 & 0.1889 & 0.0417 & 0.0264 & 0.0191 \\
0.084 & 0.132 & 0.9267 & 0.1127 & 0.0667 & 0.0442 \\
0.084 & 0.132 & 0.9267 & 0.1129 & 0.0668 & 0.0443 \\
0.142 & 0.245 & 1.0634 & 0.1757 & 0.1214 & 0.0958 \\
0.142 & 0.245 & 1.0636 & 0.1757 & 0.1215 & 0.0959 \\
\hline
\end{tabular}

Electron repulsion acts in the opposite direction (Table 6). A $10 \%$ reduction of the Racah parameters leads to a $15 \%$ larger splitting. However, unlike the excited states, the ground state seems less sensitive to the exact intensity of the electron-electron repulsion than to the ligand field and spin-orbit terms.

It is easily seen that the SO and ER parameters can affect the ground state splitting in opposite directions. However, a more complex interplay of the SO coupling constant and the three ER parameters is apparent in our full ab initio calculation. The lower SO coupling constant compared with the experimental parameters should lead to a smaller splitting, but the final splitting is one order of magnitude larger than with Carnall's SO and ER parameters. Our linear variation of the ER parameters cannot reproduce such a behavior, and it is not clear which parameters $\left(\xi, G_{2}, G_{4}\right.$ or $\left.G_{6}\right)$ plays the most significant role in this large splitting.

Obviously the intensity of the ligand field plays a generally positive role in the magnitude of the effect since no splitting occurs without it (free ion case). Nevertheless, we observe that the influence of the ligand field is not monotonous (Table 7).

The calculated X-band EPR peak-to-peak width $(26 \mathrm{G})$ is too small by one order of magnitude $(150-700$ $\mathrm{G}$ depending on the temperature in aqueous solutions of $\left.\mathrm{Gd}^{3+}[2]\right)$. Since the electron spin relaxation rates depend on the square of the magnitude parameters $a_{k}$, any discrepancy in the ground state splitting is amplified in the calculation of the EPR line width. One could also imagine that the assumption of a dominant second order term in the ZFS Hamiltonian is not valid for the $\mathrm{Gd}^{3+}$ aqua ion. Indeed, the analysis of EPR spectra in aqueous solution shows important fourth and sixth order contributions [2,27], which are marginal in our results (Table 4). Finally, our calculations are based on a gas phase optimized structure, which could differ from the true structure in solution. The so-called static ZFS contribution to relaxation represents the effect of rotational diffusion on the fine structure associated with the time-averaged coordination polyhedron. At a finite temperature, fluctuations of the structure will also take place, due to vibrations and collisions solvent molecules. These time-dependent structural fluctuations are the origin of the transient ZFS. If our ideal $\mathrm{D}_{4 \mathrm{~d}}$ symmetry does not correspond to the average structure, the theoretical ZFS is not directly comparable to the static ZFS in solution. A complete study will require a full geometry optimization without symmetry constraints, as well as a systematic exploration of the potential energy surface. Solvation effects on the optimized structure may also be important.

Table 7

Influence of the ligand field magnitude on the ZFS

\begin{tabular}{|c|c|c|c|c|c|c|c|c|}
\hline Reference & Bleaney & $0.5 \mathrm{LF}$ & $0.9 \mathrm{LF}$ & $1.1 \mathrm{LF}$ & $1.5 \mathrm{LF}$ & $1.6 \mathrm{LF}$ & $1.8 \mathrm{LF}$ & $2 \mathrm{LF}$ \\
\hline 0.000 & 0 & 0.0000 & 0.0000 & 0.0000 & 0.0000 & 0.0000 & 0.0000 & 0.0000 \\
\hline 0.000 & 0 & 0.0001 & 0.0001 & 0.0001 & 0.0000 & 0.0000 & 0.0000 & 0.0000 \\
\hline 0.032 & 0.049 & 0.0344 & 0.0348 & 0.0283 & 0.0441 & 0.0740 & 0.1433 & 0.2253 \\
\hline 0.032 & 0.049 & 0.0344 & 0.0348 & 0.0284 & 0.0442 & 0.0741 & 0.1434 & 0.2254 \\
\hline 0.084 & 0.132 & 0.0922 & 0.0919 & 0.0721 & 0.0510 & 0.1017 & 0.2148 & 0.3494 \\
\hline 0.084 & 0.132 & 0.0923 & 0.0920 & 0.0722 & 0.0511 & 0.1017 & 0.2149 & 0.3494 \\
\hline 0.142 & 0.245 & 0.1738 & 0.1611 & 0.1162 & 0.0531 & 0.1089 & 0.2440 & 0.4050 \\
\hline 0.142 & 0.245 & 0.1738 & 0.1612 & 0.1162 & 0.0531 & 0.1090 & 0.2441 & 0.4051 \\
\hline
\end{tabular}




\section{Conclusion}

We have developed a general method for the ab initio study of the electronic fine structure of lanthanide(III) complexes. This versatile method involves a configuration interaction calculation within the complete active space of the $4 \mathrm{f}^{n}$ configurations, based on a limited number of parameters that can be either calculated quantum mechanically or extracted from experimental results.

We implemented our method in a computer program. The approach proved to be quite affordable using the currently available hardware. We applied it for the determination of the ${ }^{8} S_{7 / 2}$ ground state splitting (ZFS) of the $\left[\mathrm{Gd}\left(\mathrm{H}_{2} \mathrm{O}\right)_{8}\right]^{3+}$ aqua ion. Ligand field parameters were derived from a DFT calculation, and spin-orbit and electron repulsion parameters were obtained either from the literature or from an ab initio calculation. We used our results to calculate the X-band EPR peak-topeak width in solution, based on a model of rotational modulation.

When experimental parameters are used for the spinorbit coupling and electron repulsion, the calculated ZFS magnitude agrees with the experimental EPR data in the solid state and in solution. However, the full nonempirical calculation underestimates the effect somehow. The error is more severe for the EPR peak-to-peak width that is one order of magnitude lower than the observed value. We used our program to study the influence of the various Hamiltonian contributions (electrostatic repulsion, spin-orbit coupling and ligand field) on the final ground state splitting. We observed that the spin-orbit coupling constant had a positive correlation with the ZFS, whereas increasing the electrostatic repulsion (Racah/Slater-Condon parameters) decreases the ZFS. It is more difficult to discuss the correlation between the ligand field and the ZFS as our analysis shows a non-monotonous behavior.

Although the calculated spin-orbit coupling constant and Racah parameters were similar to the reported experimental values, the full ab initio parameters (SO, ER and LF) lead to a significant overestimation of the ground state splitting.

With the availability of our fine structure calculation program, various future developments can be envisaged using the general framework presented here. A full quantum mechanical calculation of the electrostatic, ligand field and spin-orbit parameters obviously improves the predictive value of the method. Since our first results in this respect were not accurate enough, more work is required in order to assess the suitability of different theoretical methods and basis sets for our purpose. The addition of further terms (for example the interaction with higher excited configurations) can be necessary for the accurate treatment of some systems, eg., $\mathrm{Eu}^{2+}$. Using a perturbative approach would allow such an improvement without a dramatic increase of the computational cost. In order to better compare our results with the experimental data, a convenient way to project our eigenstates onto the $\left|S, m_{S}\right\rangle$ basis set used in the spin Hamiltonian formalism will also be necessary for future studies.

\section{Acknowledgements}

This project was carried out in the framework of the EC COST D9 action 'Modeling of increasingly complex systems'. We also thank the Swiss National Science Foundation, the University of Lausanne and the Swiss Federal Institute of Technology for financial support.

\section{References}

[1] A.E. Merbach, É. Tóth, The Chemistry of Contrast Agents in Medical Magnetic Resonance Imaging, Wiley, Chichester, UK, 2001.

[2] S. Rast, A. Borel, L. Helm, E. Belorizky, P.H. Fries, A.E. Merbach, J. Am. Chem. Soc. 123 (2001) 2637.

[3] S. Rast, P.H. Fries, E. Belorizky, J. Chem. Phys. 113 (2000) 8724.

[4] A. Abragam, B. Bleaney, Electron Paramagnetic Resonance of Transition Ions, Oxford University Press, Oxford, 1970, p. 335.

[5] H.A. Buckmaster, R. Chatterjee, Y.H. Shing, Can. J. Phys. 50 (1972) 991.

[6] M.R. Smith, Y.H. Shing, R. Chatterjee, H.A. Buckmaster, J. Magn. Res. 36 (1977) 351.

[7] C. Görller-Walrand, K. Binnemans, in: K.A.J. Gschneider, L. Eyring (Eds.), Handbook on the Physics and Chemistry of Rare Earths, Elsevier, New York, 1996, p. 121.

[8] C.E. Schäffer, C.K. Jørgensen, Mol. Phys. 9 (1965) 401.

[9] C.K. Jørgensen, J. Phys. F 26 (1965) 825.

[10] D.J. Newman, B. Ng, Rep. Prog. Phys. 52 (1989) 699

[11] C. Rudowicz, H.W.F. Sung, Physica B 300 (2001) 1.

[12] J.C. Slater, Phys. Rev. 35 (1929) 1293.

[13] E.U. Condon, Phys. Rev. 36 (1930) 1121.

[14] E.U. Condon, G.H. Shortley, The Theory of Atomic Spectra, University Press, Cambridge, 1970, p. 178.

[15] G.H. Dieke, Spectra and Energy Levels of Rare Earths Ions in Crystals, Wiley, New York, 1968.

[16] A.J. Freeman, R.E. Watson, Phys. Rev. 127 (1962) 2058.

[17] W.T. Carnall, P.R. Fields, K. Rajnak, J. Chem. Phys. 49 (1968) 4443.

[18] M. Atanasov, C.A. Daul, C. Rauzy, Chem. Phys. Lett. 367 (2003) 737.

[19] M. Atanasov, C.A. Daul, C. Rauzy, Structure and Bonding (in press).

[20] Matla в, The Mathworks, Inc., 2000.

[21] E.J. Baerends, Amsterdam Density Functional, Scientific Computing and Modelling, Amsterdam, 2001.

[22] O. Schafer, C. Daul, Int. J. Quant. Chem. 61 (1997) 541.

[23] A.D. Becke, J. Chem. Phys. 85 (1986) 7184

[24] J. Perdew, Phys. Rev. B 33 (1986) 8822.

[25] M. Atanasov, C. Rauzy, private communication.

[26] C. Daul, ХАтом.

[27] A. Borel, F. Yerly, L. Helm, A.E. Merbach, J. Am. Chem. Soc. 124 (2002) 2042.

[28] H.A. Buckmaster, R. Chatterjee, Y.H. Shing, Phys. Stat. Sol. (a) 13 (1972) 9. 
[29] T. Kurisaki, T. Yamaguchi, H. Wakita, J. Alloy. Compd. 192 (1993) 293.

[30] S. Hengrasmee, M.M. Probst, Z. Naturforsch. 46a (1991) 117.

[31] K. Rajnak, J. Chem. Phys. 43 (1965) 847.
[32] K. Rajnak, B.G. Wybourne, J. Chem. Phys. 41 (1964) 565.

[33] K. Rajnak, B.G. Wybourne, Phys. Rev. 132 (1963) 280.

[34] B.G. Wybourne, Phys. Rev. 148 (1966) 317. 